\title{
ROL DEL SEGUIMIENTO ULTRASONOGRÁFICO EN LA TOMA DE DECISIONES EN NIÑOS CON SOSPECHA DE ABDOMEN AGUDO DE ORIGEN APENDICULAR
} \author{
Martin $\boldsymbol{P}^{(3)}$. \\ 1. Diagnóstico por Imágenes. Clínica las Condes, Santiago, Chile. \\ 2. Cirugía Infantil, Clínica las Condes, Santiago, Chile. \\ 3. Becado de Pediatría, Hospital Roberto del Río, U de Chile. Santiago, Chile.
}

Drs. Ximena Ortega $F^{(1)}$, Karla Moënne $B^{(1)}$, Juan Antonio Escaffi ${ }^{(1)}$, Carolina Pérez $\mathbf{S}^{(1)}$, Jorge Godoy $L^{(2)}$,

Rodrigo

\begin{abstract}
Patients with unspecific acute abdominal pain and patients with nonspecific acute abdominal pain and nonconclusive physical examination for appendicitis are usually kept under medical control. In our Emergency Department some pediatricians and surgeons have added a second ultrasound (US) examination to clinical follow-up in patients with low suspicion of appendicitis and discordant or nonconclusive initial findings. The objective of this study was to evaluate the role of a second US scan in medical or surgical treatment. We retrospectively evaluated medical records (from November 2006 to June 2008) of 1.959 patients with a history of acute abdominal pain referred for abdominal US examination. Fifty-four patients, 22 males and 32 females aged between 3 and 14 years, received clinical indication for a second US study during that period. US monitoring was performed between 5 to 36 hours after the first examination. Patient history details, ultrasonographic characterization of cecal appendix, and clinical evolution were registered. Ten patients had progressive inflammatory appendicular changes. All of them underwent surgery and appendicitis was confirmed in eight cases (80\%). Ten patients showed no changes on the second US scan; $40 \%$ of patients underwent surgery with biopsies confirming lymphoid hyperplasia (2), and congestive changes (2). Fifteen patients had a second US study that showed regression of inflammatory changes; all of them received medical treatment. In 19 patients comparison was not feasible since appendixes appeared normal
\end{abstract}

Ortega $X$. Rol del seguimiento ultrasonográfico en la toma de decisiones en niños con sospecha de abdomen agudo de origen apendicular. Rev Chil Radiol 2010; 16(4): 200-204. Correspondencia: Dra. Ximena Ortega F. xortegaf@gmail.com

Trabajo recibido el 25 de octubre de 2010, aceptado para publicación el 23 de noviembre de 2010. on US examination, or due to insufficient visualization of appendix. Our results suggest that ultrasound follow-up promotes adequate decision-making when facing surgical or medical treatment options for pediatric patients presenting with abdominal pain with initial clinical and imaging findings inconclusive for acute appendicitis.

Key words: Acute Appendicitis, Spontaneous resolution, Ultrasound.

Resumen: Pacientes con dolor abdominal no específico o examen físico no concluyente para apendicitis aguda son usualmente mantenidos en control médico. En nuestro Sevicio de Urgencia algunos pediatras y cirujanos agregan una ultrasonografía (US) de control en la evolución de cuadros no concluyentes. El objetivo de este trabajo fue evaluar el rol de este segundo US en la toma de decisiones médicas o quirúrgicas. Para esto evaluamos retrospectivamente las fichas de 1959 pacientes referidos del Servicio de Urgencia para US abdominal con historia de dolor abdominal entre noviembre del 2006 y junio del 2008. Cincuenta y cuatro pacientes, 22 niños y 32 niñas entre 3 y 14 años recibieron durante ese período indicación de un segundo US. Estos exámenes fueron realizados entre 5 y 36 horas luego del primer estudio ultrasonográfico. Se registraron datos relacionados a la historia, caracterización ultrasongráfica del apéndice y evolución clínica. Diez pacientes tuvieron cambios inflamatorios progresivos. Todos fueron a cirugía y la apendicitis fue confirmada en ocho casos (80\%). Diez pacientes tuvieron cambios inflamatorios que no se modificaron en el segundo US; $40 \%$ fue a cirugía con biopsias que demostraron hiperplasia linfoide (2) y cambios congestivos (2). Quince pacientes mostraron regresión de los cambios inflamatorios en el segundo US; todos recibieron tratamiento médico. En 19 pacientes la comparación no fue posible, por tratarse de apéndices ecográficamente normales o debido 
a insuficiente visualización del apéndice. Nuestros resultados sugieren que el seguimiento ecográfico ayuda en la decisión de tratamiento médico o quirúrgico en pacientes que se presentan con dolor abdominal cuyos hallazgos clínicos e imaginológicos iniciales no son concluyentes de apendicitis aguda.

Palabras clave: Apendicitis aguda, Resolución espontánea, Ultrasonografía.

\section{Introducción}

La apendicitis aguda (AA) es la causa más frecuente de abdomen agudo en el niño. A pesar del notable progreso en las técnicas de apoyo, tanto de imágenes como de laboratorio, su diagnóstico sigue representando un constante desafío en la práctica pediátrica de urgencia. A la presentación inhabitual de la apendicitis, ya reportada en la literatura ${ }^{(1)}$, se suma la consulta precoz como un hecho creciente y la presión, tanto de padres como de médicos, para excluir el diagnóstico de AA en estos pacientes, con la mayor certeza y prontitud posible.

Los hallazgos ultrasonográficos, cuando son categóricos, indiscutiblemente apoyan al clínico en la decisión quirúrgica. Estos hallazgos incluyen: el aumento de calibre sobre $6 \mathrm{~mm}$, la ausencia de compresión a la presión del transductor y el aumento de la ecogenicidad del tejido periapendicular (Figura 1). Frente a la duda diagnóstica que se origina habitualmente por una consulta precoz o dolor abdominal que no reúne clínicamente todas las características de la $A A$, un porcentaje de estos pacientes quedan habitualmente en control clínico.

La amplia disponibilidad de la ultrasonografía (US), su bajo costo comparativo en nuestro medio y la ausencia de radiación en el examen, han hecho que algunos pediatras y cirujanos estén incorporando una segunda US al control clínico cercano.

El objetivo de nuestro trabajo fue valorar el rol de la segunda US en el seguimiento de pacientes que acudieron al servicio de urgencia con historia de dolor abdominal, en ausencia de signos clínicos y/o ultrasonográficos concluyentes de AA.

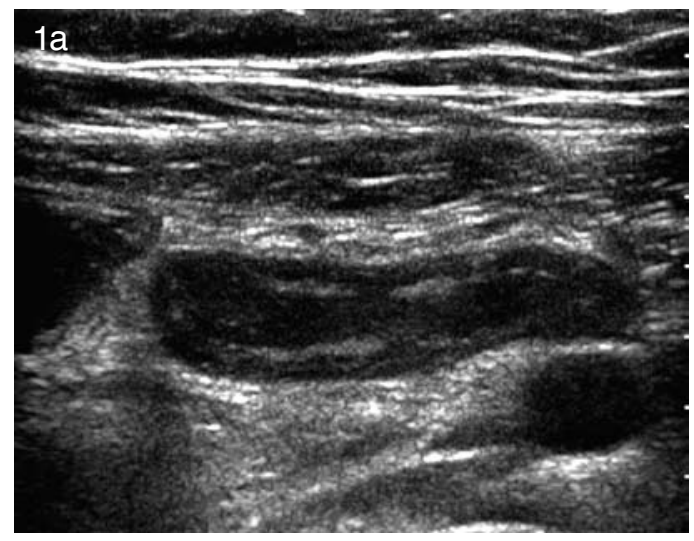

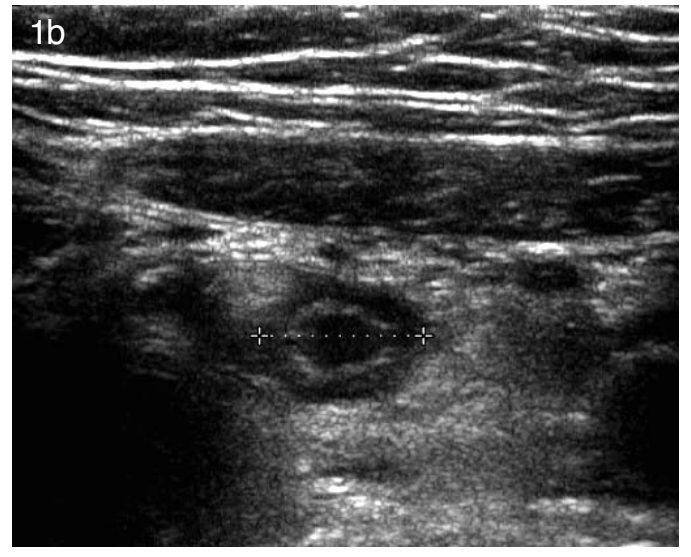

Figura 1. Apendicitis aguda en US. Apéndice no compresible, con signos inflamatorios, de calibre aumentado. (a) longitudinal, (b) transversal.

\section{Materiales y métodos}

Durante un año, en el período comprendido entre noviembre del 2006 y junio del 2008 se buscó en el sistema RIS-PAC del Servicio de Radiología de Clínica Las Condes, los pacientes provenientes del servicio de urgencia que hubieran sido ingresados para dos US abdominales durante un período no superior a 36 horas.

Se revisaron sus fichas clínicas, registrando datos sobre antecedentes mórbidos, motivo de consulta, fecha y hora de ambas US y resultados anatomopatológicos de biopsias, en aquellos pacientes que fueron sometidos a apendicectomía.

Se revisó el registro de imágenes y los informes de US efectuadas, registrando hallazgos relativos a la visualización satisfactoria del apéndice cecal y características ultrasonográficas como: calibre, vascularización de la pared, aumento de la ecogenicidad del tejido periapendicular y presencia de coprolitos en el lumen. Los exámenes fueron realizados indistintamente por médicos radiólogos pediatras y radiólogos de urgencia. Se utilizaron ecotomógrafos ALT HDI 5000 y Siemmens Antares. Se consideraron como características de apéndice normal: calibre homogéneo inferior a $6 \mathrm{~mm}$, compresible a la presión del transductor, sin aumento de la vascularización de la pared durante la exploración Doppler color ni de la ecogenicidad del tejido periapendicular y sin coprolitos (Figura 2). Estas características fueron analizadas y comparadas en ambas US, utilizando una planilla Excel 2007.

En atención a esta comparación, los hallazgos de la segunda US fueron sintetizados en:

a) Progresión: cambios inflamatorios que progresan, en aquellos casos en que al menos una de las características mostraba incremento de su magnitud 
b) Estable patológico: cambios inflamatorios estables, en aquellos apéndices cuyas alteraciones no mostraron variación significativa en la segunda US

c) Regresión: cambios inflamatorios que regresan, en aquellos donde al menos una de sus características, descrita previamente como patológica, mostró disminución de su magnitud

d) Normal: apéndice estable normal, en aquellos donde no se registraron alteraciones en ambas US

e) No clasificable: en aquellos pacientes en que no fue posible la visualización del apéndice cecal en la segunda US.
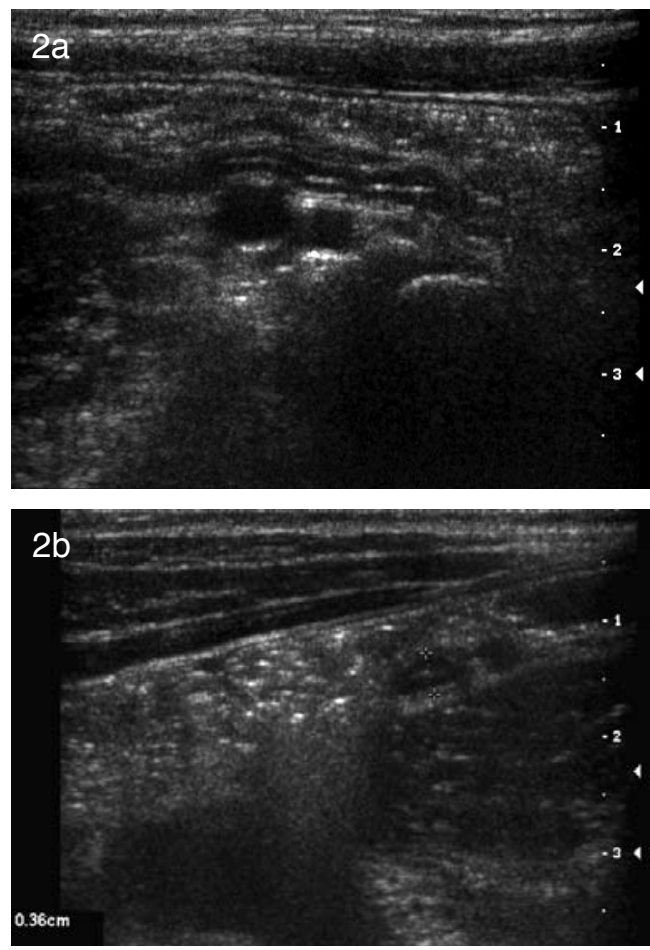

Figura 2. US muestra apéndice normal. (a) Imagen longitudinal en fosa ilíaca derecha, (b) transversal.

\section{Resultados}

Durante el período de estudio se realizaron 1.959 US a pacientes con historia de dolor abdominal, provenientes del Servicio de Urgencia. De ellos, 54 pacientes (32 niñas y 22 niños) entre 3 y 14 años fueron sometidos a una segunda US como parte del seguimiento y control de cuadros de dolor abdominal, presentes desde 2 horas a 9 días previo a la consulta. El segundo examen de US fue realizado entre 5 y 36 horas luego del inicial.

Del total de pacientes controlados, 10 presentaron signos inflamatorios que se consideraron progresivos en la segunda US (Figura 3); todos ellos fueron sometidos a apendicectomía siendo el resultado anatomopatológico de las piezas para biopsia: apendicitis fibrinonoleucocitaria en 8 casos e hiperplasia linfoide en 2 de ellos.
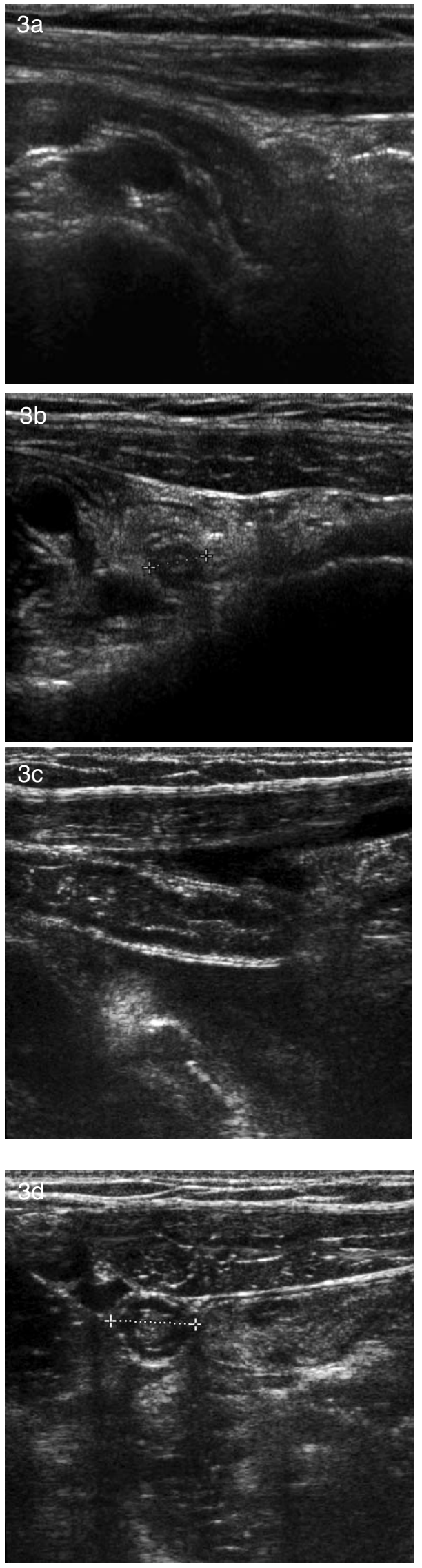

Figura 3. US: Apéndice con cambios inflamatorios $(a, b)$, que progresan en el control $(c, d)$. 
Los pacientes en los cuales se consideró retrospectivamente que las alteraciones descritas en el primer US permanecían sin cambios en el control (Figura 4) correspondieron también a 10. De ellos, 4 fueron a cirugía por decisión clínica y la biopsia reportó: existencia de hiperplasia folicular linfoide en 2 casos y de signos de congestión en los otros 2, uno de ellos con un coprolito en el lumen. Los 6 pacientes restantes fueron manejados médicamente.
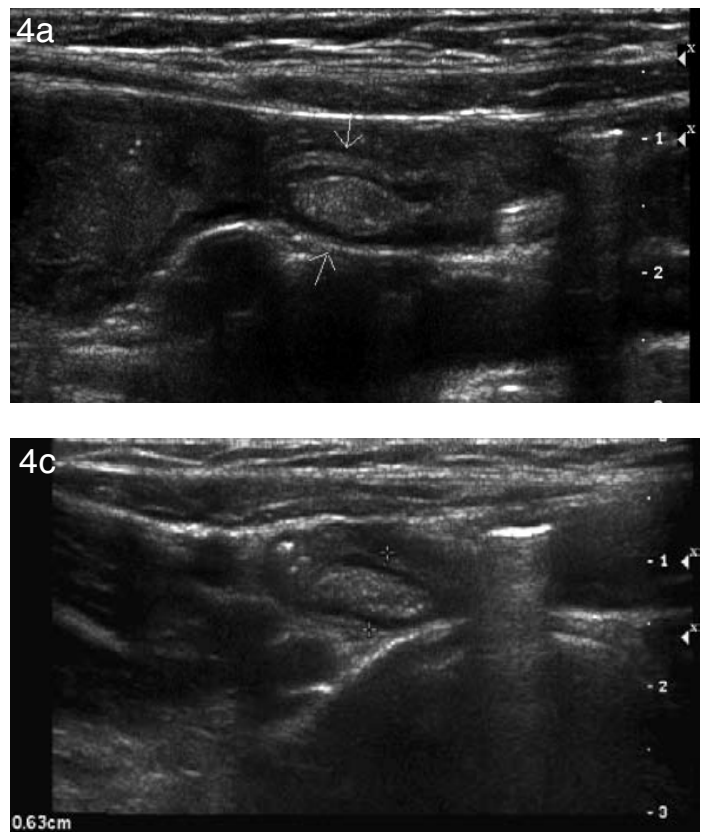

Figura 4. US: Apéndice con cambios inflamatorios estables. Apéndice no compresible, con contenido ecogénico y calibre aumentado (a,b), que mantiene sus características en control efectuado 10 horas más tarde (c,d).
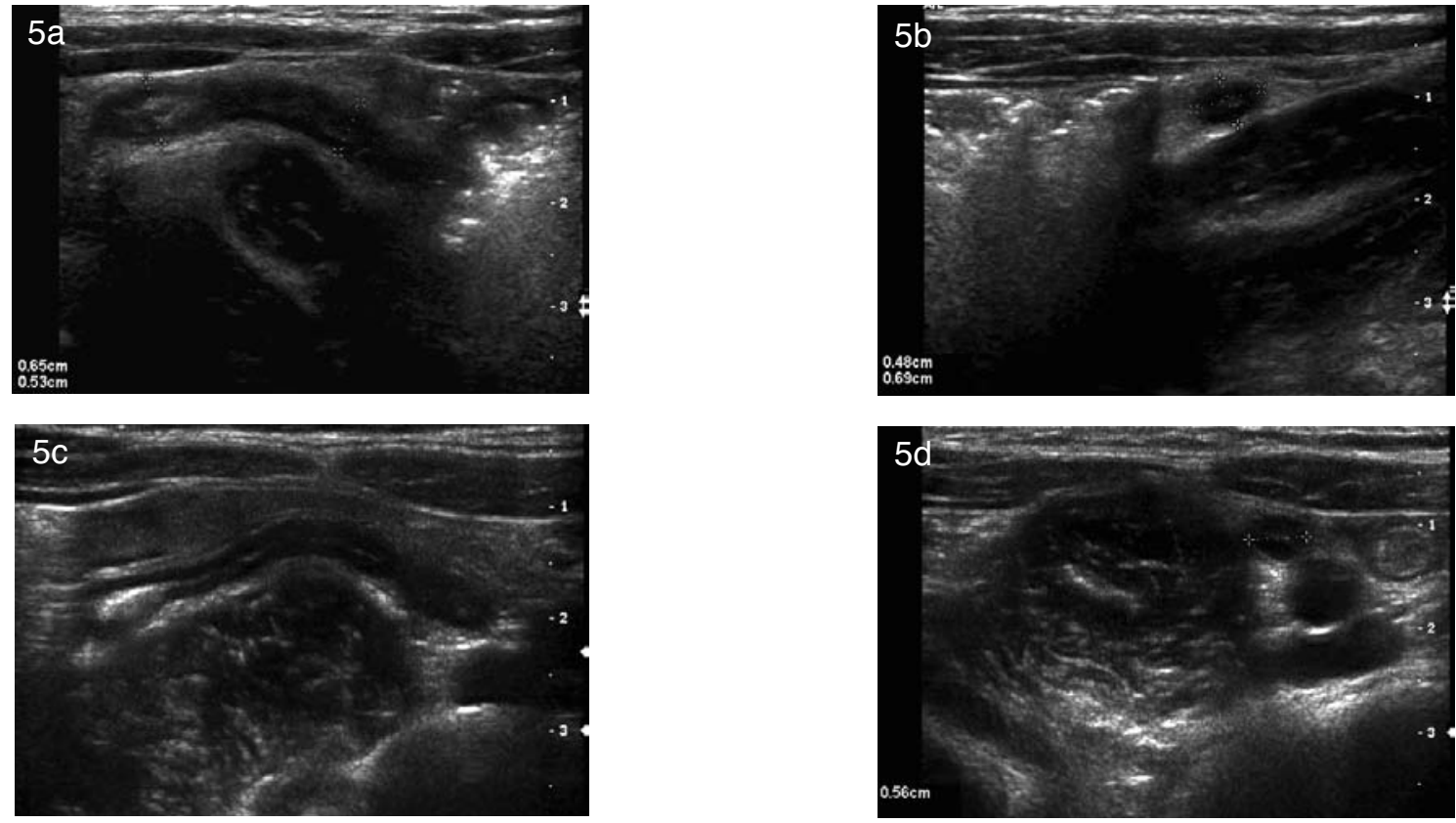

Figura 5. Cambios inflamatorios apendiculares que regresan en el control. Apéndice con engrosamiento de la punta, no compresible en toda su extensión $(a, b)$. En control efectuado 8 horas más tarde se observa disminución de su calibre y aparición de aire luminal $(c, d)$.

En 15 pacientes la comparación estableció que como mínimo uno de los parámetros considerados como patológicos en la primera US había regresado hacia su condición normal, al menos parcialmente (Figura 5). De este grupo, sólo dos pacientes fueron operados, uno de ellos con un apéndice informado como hiperplasia linfoide e infiltración fibrinoadiposa de tipo involutiva en la biopsia y el otro con un quiste del paraoóforo derecho y apéndice normal. Los 13 pacientes restantes fueron manejados médicamente.
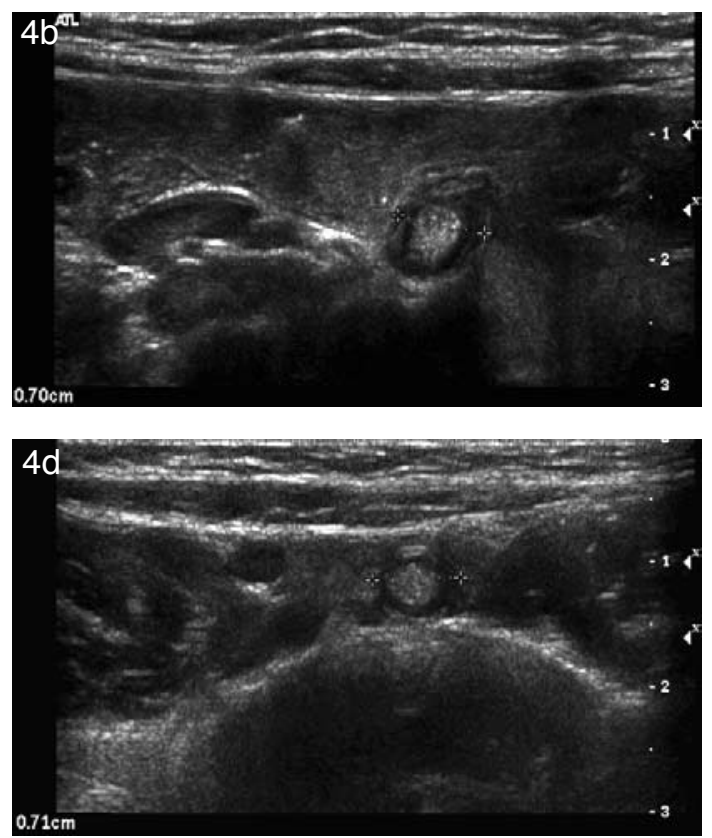
re 
En los 19 pacientes restantes no fue posible establecer una comparación de los hallazgos considerados como patológicos, por tratarse de apéndices descritos como normales en ambos exámenes (9 pacientes) 0 por la imposibilidad de visualizar el apéndice cecal en uno o ambos exámenes (10 pacientes).

\section{Discusión}

Así como ha sucedido en muchas otras patologías, el cuadro clínico de la AA ha sido modelado en adultos y traspasado a los niños. Sin embargo, tanto la presentación como la evolución de la apendicitis en niños pueden ser inhabituales, con características atípicas que dificultan el diagnóstico(1).

Esta situación es compartida en el diagnóstico por US donde, con alguna frecuencia, se presentan pacientes con apéndices que no son normales pero que no reúnen criterios suficientes para catalogarlos como AA; con frecuencia, los hallazgos en estos pacientes son reportados como no concluyentes 0 simplemente normales.

La conexión con el resto del tubo digestivo hace que el apéndice participe en otros procesos del abdomen, respondiendo con cambios inflamatorios que no siempre terminan en obstrucción y perforación ${ }^{(2)}$. Establecer entonces qué pacientes corresponden a una $\mathrm{AA}$ en evolución, a diferencia de aquellos con cambios inflamatorios secundarios o transitorios es un desafío permanente para el clínico, donde la US ofrece un apoyo que, en nuestra opinión, no ha sido completamente valorado. La literatura reporta los cambios obtenidos en el manejo de pacientes con intervención de la tomografía computada ${ }^{(3,4)}$, cuyo uso implica una dosis de radiación que pudiera evitarse haciendo buen uso de la US, trabajando en equipo con los clínicos ${ }^{(5)}$. Los resultados obtenidos en el grupo de pacientes cuyos estudios demostraron progresión de los cambios inflamatorios, da cuenta de que es posible contribuir con la US a distinguir aquellos pacientes que padecen un proceso inflamatorio del apéndice que requerirá de intervención quirúrgica.

El control clínico de pacientes con cuadros de dolor abdominal poco claros y sospecha de AA representa una práctica frecuente, ampliamente aceptada.
La conexión que se establece con el paciente durante la primera consulta permite la observación cercana de los cambios clínicos en condiciones seguras, no aumentando el riesgo de perforación ${ }^{(2,6)}$. La adición de la US como control, debiera ser expresión de los cambios que pudiera experimentar el apéndice durante este intervalo.

Con este objetivo, nos ha parecido importante establecer una nomenclatura que permita transmitir al clínico en forma eficiente nuestra impresión de las características del apéndice, como parte de un proceso evolutivo, y de qué manera estos elementos acercan o alejan la posibilidad de una AA en curso, justificando una intervención quirúrgica. La terminología mencionada en este reporte, utilizada para referirse al apéndice en la US de control, ha sido incorporada a nuestro informe rutinario de los exámenes de aquellos pacientes que son controlados por sospecha de AA y constituye una herramienta efectiva en la toma de decisiones para el cirujano infantil y pediatra a cargo del paciente.

\section{Bibliografía}

1. Becker T, Kharbanda A, Bachur R. Atypical clinical features of pediatric appendicitis. Acad Emerg Med 2007; 14(2): 124-129. Epub 2006 Dec 27.

2. Andersson RE. The natural history and traditional management of appendicitis revisited: spontaneous resolution and predominance of prehospital perforations imply that a correct diagnosis is more important than an early diagnosis. World J Surg 2007; 31: 86-92.

3. Hagendorf BA, Clarke JR, Burd RS. The optimal initial management of children with suspected appendicitis: a decision analysis. J Pediatr Surg 2004; 39(6): 880885.

4. Stephen AE, Segev DL, Ryan DP, Mullins ME, Kim SH, Schnitzer JJ et al. The diagnosis of acute appendicitis in a pediatric population: to CT or not to CT. J Pediatr Surg 2003; 38(3): 367-371.

5. Toorenvliet BR, Wiersma F, Bakker RF, Merkus JW, Breslau PJ, Hamming JF. Routine ultrasound and limited computed tomography for the diagnosis of acute appendicitis. World J Surg 2010; 34(10): 2278-2285.

6. Colson $M$, Skinner KA, Dunnington $G$. High negative appendectomy rates are no longer acceptable. Am J Surg 1997; 174: 723-726. 